\title{
Reconstruction method in the kinetic gravity braiding theory with shift-symmetric
}

\author{
Ruslan K. Muharlyamov ${ }^{1, *}$ and Tatiana N. Pankratyeva ${ }^{2, \dagger}$ \\ ${ }^{1}$ Department of General Relativity and Gravitation, Institute of Physics, \\ Kazan Federal University, Kremlevskaya str. 18, Kazan 420008, Russia \\ ${ }^{2}$ Department of Higher Mathematics, Kazan State Power Engineering \\ University, Krasnoselskaya str. 51, Kazan 420066, Russia
}

\begin{abstract}
We present a reconstruction method for flat Friedman-Robertson-Walker (FRW) spacetime in a subclass of Horndeski theory - specifically shiftsymmetric, the kinetic gravity braiding (KGB) theory with a non-vanishing conserved current. Choosing the form of the Hubble parameter and kinetic density $X$, we restore the functions $G_{2}, G_{3}$ of the KGB model. In order to determine whether the model is free of ghosts and Laplacian instabilities and thus cosmologically viable, two conditions related to scalar perturbations are checked. Initially, the Lagrangian does not include the term that describes, for example, the perfect fluid with the EoS parameter $w \neq-1$. This fluid can provide a dynamic solution $H(t), X(t)$. In the presented method, dynamic solutions are provided by a nonzero scalar charge associated with the shift symmetry $\phi \rightarrow \phi+\phi_{0}$. Reconstruction examples are given for models: an perfect fluid, a unified description dark energy-dark matter, a post-inflationary transition to the radiation-dominated phase.
\end{abstract}

PACS numbers: $04.50 . \mathrm{Kd}$

Keywords: Horndeski theory; kinetic gravity braiding theory; dark energy theory; cosmological perturbation theory

\section{INTRODUCTION}

General relativity (GR) is confirmed by many observational data [1 [5]. But to explain the accelerated expansion of the Universe and other observational facts, within the framework of GR one has to involve the hypothesis of the existence of an exotic substance called the relativistic dark energy (DE). An alternative solution is to construct modified gravity models. Also, an important motive for modifying the gravity theory is the impossibility of extrapolating GR to all scales. There is a well-known problem of compatibility of GR with quantum mechanics [6 6 9 ].

The Horndeski gravity (HG) occupies a special place among the modified models [10. The field equations in GR are differential equations of the second order, thus, evading Ostrogradski instabilities arising [11, 12. The HG is the most general variant of the scalar-tensor theory of gravitation with motion equations of the second order. The HG includes, as special cases, all known examples of scalar-tensor gravitational theories of the second order (Brans-Dicke theory, $f(R)$ gravity, quintessence, etc.) [13 18. The action density of HG contains several functions that provide a broad phenomenology. This makes it possible to solve important cosmological and astrophysical problems (screening of the cosmological constant, kinetic inflation, late de Sitter stage, hairy black holes, etc.) [19 23].

Various criteria for the selection of alternative gravity theories and physical models proposed by them are discussed. In HG the derivative self-couplings of the scalar field screen the deviations from GR at small scales or high densities through the Vainshtein mechanism 24], thus satisfying solar system and early universe constraints 25, 26]. After the gravitational wave event GW170817 [5], the modified theories of gravity, in which the propagation speed of gravitational waves can differ from the speed of light, have a dubious status [27, 28. When considering modified models, one has to worry about the presence of ghosts as well as Laplacian instabilities [29, 30]. In spite of these constraints, the HG has retained most of the viable models.

The HG is determined by the following action density

$$
L_{H}=\sqrt{-g}\left(\mathcal{L}_{2}+\mathcal{L}_{3}+\mathcal{L}_{4}+\mathcal{L}_{5}\right),
$$

one has (in the parameterization of ref. [31]):

$$
\mathcal{L}_{2}=G_{2}(\phi, X), \mathcal{L}_{3}=-G_{3}(\phi, X) \square \phi,
$$

*Electronic address: rmukhar@mail.ru

${ }^{\dagger}$ Electronic address: ghjkl.15@list.ru 


$$
\begin{gathered}
\mathcal{L}_{4}=G_{4}(\phi, X) R+G_{4 X}(\phi, X)\left[(\square \phi)^{2}-\left(\nabla_{\mu} \nabla_{\nu} \phi\right)^{2}\right], \\
\mathcal{L}_{5}=G_{5}(\phi, X) G_{\mu \nu} \nabla^{\mu} \nabla^{\nu} \phi-\frac{1}{6} G_{5 X}\left[(\square \phi)^{3}-3 \square \phi\left(\nabla_{\mu} \nabla_{\nu} \phi\right)^{2}+2\left(\nabla_{\mu} \nabla_{\nu} \phi\right)^{3}\right],
\end{gathered}
$$

respectively, where $g$ is the determinant of metric tensor $g_{\mu \nu} ; R$ is the Ricci scalar and $G_{\mu \nu}$ is the Einstein tensor; the factors $G_{i}(i=2,3,4,5)$ are arbitrary functions of the scalar field $\phi$ and the canonical kinetic term, $X=-\frac{1}{2} \nabla^{\mu} \phi \nabla_{\mu} \phi$. We consider the definitions $G_{i X} \equiv \partial G_{i} / \partial X,\left(\nabla_{\mu} \nabla_{\nu} \phi\right)^{2} \equiv \nabla_{\mu} \nabla_{\nu} \phi \nabla^{\nu} \nabla^{\mu} \phi$, and $\left(\nabla_{\mu} \nabla_{\nu} \phi\right)^{3} \equiv \nabla_{\mu} \nabla_{\nu} \phi \nabla^{\nu} \nabla^{\rho} \phi \nabla_{\rho} \phi \nabla^{\mu} \phi$.

We will consider the KGB theory: $G_{2} \neq 0, G_{3} \neq 0, G_{4}=1 /(16 \pi), G_{5}=0$. The shift-symmetric sector of the theory corresponds to the choice $G_{i}=G_{i}(X)$. Then the action density (1) takes the form

$$
L_{H}=\sqrt{-g}\left(\frac{R}{16 \pi}+G_{2}(X)-G_{3}(X) \square \phi\right) .
$$

This theory is not sensitive to the constraints associated with the gravitational event GW170817. The KGB cosmologies have been extensively studied in the past, for example in [34. In these models, the Universe smoothly evolves from contraction to expansion, suffering neither from ghosts nor Laplacian instabilities around the turning point. The end-point of the evolution can be a standard radiation-domination era or an inflationary phase. The necessary constraints for Lagrangians are formulated, which are necessary to obtain a healthy bounce. In the work [35], authors analyze what happens when the Horndeski Lagrangian is varied within the Palatini approach by considering the metric and connection as independent variables. For example, in the context of the connection with the Palatini approach, it was shown that the KGB subset of the HG cosmologies may not contain ghosts.

We present a reconstruction method for flat Friedman-Robertson-Walker (FRW) spacetimes in the subclass of HG (3) with a non-vanishing conserved current. Choosing the form of the Hubble parameter $H$ and kinetic density $X$, we restore the functions $G_{2}$ and $G_{3}$. Next, we check if the model is free of ghosts and Laplacian instabilities and thus cosmologically viable. This method allows for a realistic model of the Universe to simply reconstruct some field theory. The work [32] considers reconstruction in the KGB theory with the vanishing conserved current and the shiftsymmetric. In this theory, the dynamics in time of parameters $H(t)$ and $X(t)$ is provided by the presence of other matter with variable density $\rho(t)$. We are considering a model that contains only a scalar field $\phi$ as source. The dynamism of functions $H(t)$ and $X(t)$ is provided by the non-vanishing conserved current. For example, we will reconstruct theories for models: an perfect fluid, a unified description dark energy-dark matter, a post-inflationary transition to the radiation-dominated phase.

\section{FIELD EQUATIONS}

We consider a spatially-flat FRW metric

$$
d s^{2}=-d t^{2}+a^{2}(t)\left(d x^{2}+d y^{2}+d z^{2}\right) .
$$

Denoting $H=\dot{a} / a$ the Hubble parameter, the non-trivial gravitational equations for (3) are

$$
\begin{gathered}
\frac{3 H^{2}}{8 \pi}=-G_{2}+\dot{\phi}^{2} G_{2 X}+3 H \dot{\phi}^{3} G_{3 X} \equiv \rho_{\phi}, \\
\frac{-2 \dot{H}-3 H^{2}}{8 \pi}=G_{2}-G_{3 X} \ddot{\phi} \dot{\phi}^{2} \equiv p_{\phi},
\end{gathered}
$$

where $\rho_{\phi}$ is the energy density and $p_{\phi}$ is the pressure of the scalar.

The scalar equation

$$
\nabla_{\mu}\left(-\nabla^{\mu} \phi\left[G_{2 X}-G_{3 X} \square \phi\right]+G_{3 X} \nabla^{\mu} X\right)=0
$$

has the structure of current conservation, due to the theory invariance under shifts $\phi \rightarrow \phi+\phi_{0}$. In the FRW space, the scalar current

$$
J^{\mu} \equiv-\nabla^{\mu} \phi\left[G_{2 X}-G_{3 X} \square \phi\right]+G_{3 X} \nabla^{\mu} X
$$

reduces to

$$
J^{\mu}=\delta_{0}^{\mu} \dot{\phi}\left(G_{2 X}+3 H G_{3 X} \dot{\phi}\right)
$$


The scalar field equation (7) becomes

$$
\frac{1}{a^{3}} \frac{d}{d t}\left[a^{3} \dot{\phi}\left(G_{2 X}+3 H G_{3 X} \dot{\phi}\right)\right]=0
$$

It has the first integral

$$
\dot{\phi}\left(G_{2 X}+3 H G_{3 X} \dot{\phi}\right)=\frac{C_{0}}{a^{3}},
$$

where $C_{0}$ is the scalar charge associated with the shift symmetry $\phi \rightarrow \phi+\phi_{0}$. Among equations (5), (6) and (11) only two are independent. We have four functions $\left(H(a), X(a), G_{2}, G_{3}\right)$ and two independent equations. Therefore, we have two degrees of freedom. By setting the expansion scenario of the Universe $H(a)$ and the kinetic dependence $X(a)$, we find the functions $G_{2}(X)$ and $G_{3}(X)$. We will note an important property of the presented model. If the right-hand side of equation (11) is zero, then the system (5), (6), (11) can only have a stationary solution $H, X=$ const. To provide a broad variety of cosmological scenarios, we will consider the model with the non-vanishing scalar current, i.e $C_{0} \neq 0$ and $\dot{\phi} \neq 0$.

Combining the equations (5), (6) and (11), we arrive at the equalities

$$
\begin{gathered}
G_{2}=-\frac{3 H^{2}[a(X)]}{8 \pi}+\frac{\varepsilon C_{0} \sqrt{2 X}}{a^{3}(X)}, \\
G_{3 X}=\frac{H_{X}^{\prime}}{4 \pi \varepsilon \sqrt{2 X}}+\frac{C_{0} a_{X}^{\prime}}{H a^{4}(X)},
\end{gathered}
$$

where $\varepsilon= \pm 1$ defines the sign of the derivative $\dot{\phi}=\varepsilon \sqrt{2 X}$. Here we took into account $\dot{\phi} \ddot{\phi}=\dot{X}=a H / a_{X}^{\prime}$. It is assumed that for the given function $X(a)$ there is an inverse function $a(X)$.

It is clear that the Hubble parameter $H(a)$ is set from cosmological considerations. What will determine the choice of kinetic density $X(a)$ ? This arbitrariness will be used to ensure the viability of the model. By choosing the function $X(a)$, we will exclude ghosts and Laplacian instabilities in the region of applicability of the $H(a)$ model. Thus, two conditions related to scalar perturbations must be satisfied [29]:

$$
c_{S}^{2} \equiv \frac{3\left(2 w_{1}^{2} w_{2} H-w_{2}^{2} w_{4}+4 w_{1} w_{2} \dot{w}_{1}-2 w_{1}^{2} \dot{w}_{2}\right)}{w_{1}\left(4 w_{1} w_{3}+9 w_{2}^{2}\right)} \geq 0
$$

for the avoidance of Laplacian instabilities associated with the scalar field propagation speed, and

$$
Q_{S} \equiv \frac{w_{1}\left(4 w_{1} w_{3}+9 w_{2}^{2}\right)}{3 w_{2}^{2}}>0
$$

for the absence of ghosts, where

$$
\begin{aligned}
w_{1} \equiv & 2\left(G_{4}-2 X G_{4 X}\right)-2 X\left(G_{5 X} \dot{\phi} H-G_{5 \phi}\right) \\
w_{2} \equiv & -2 G_{3 X} X \dot{\phi}+4 G_{4} H-16 X^{2} G_{4 X X} H+4\left(\dot{\phi} G_{4 \phi X}-4 H G_{4 X}\right) X+2 G_{4 \phi} \dot{\phi} \\
& +8 X^{2} H G_{5 \phi X}+2 H X\left(6 G_{5 \phi}-5 G_{5 X} \dot{\phi} H\right)-4 G_{5 X X} \dot{\phi} X^{2} H^{2}, \\
w_{3} \equiv & 3 X\left(G_{2 X}+2 X G_{2 X X}\right)+6 X\left(3 X \dot{\phi} H G_{3 X X}-G_{3 \phi X} X-G_{3 \phi}+6 H \dot{\phi} G_{3 X}\right) \\
& +18 H\left(4 H X^{3} G_{4 X X X}-H G_{4}-5 X \dot{\phi} G_{4 \phi X}-G_{4 \phi} \dot{\phi}+7 H G_{4 X} X+16 H X^{2} G_{4 X X}-2 X^{2} \dot{\phi} G_{4 \phi X X}\right) \\
& +6 H^{2} X\left(2 H \dot{\phi} G_{5 X X X} X^{2}-6 X^{2} G_{5 \phi X X}+13 X H \dot{\phi} G_{5 X X}-27 G_{5 \phi X} X+15 H \dot{\phi} G_{5 X}-18 G_{5 \phi}\right), \\
w_{4} \equiv & 2 G_{4}-2 X G_{5 \phi}-2 X G_{5 X} \ddot{\phi} .
\end{aligned}
$$

For the tensor perturbations the conditions for avoidance of ghost and Laplacian instabilities are respectively written as 29

$$
Q_{T} \equiv \frac{w_{1}}{4}>0, c_{T}^{2} \equiv \frac{w_{4}}{w_{1}} \geq 0
$$

Conditions $(20)$ are satisfied for the model under consideration: $Q_{T}=1 /(32 \pi)>0, c_{T}^{2}=1>0$. Taking into account 12 and 13), we rewrite functions $c_{S}^{2}$ and $Q_{S}$ in the form

$$
Q_{S}=-\frac{3 B X^{3 / 2}}{4 \pi a^{4} H X_{a}^{\prime} w_{2}}=\frac{3 B X^{3 / 2}}{2 \pi a^{4} H^{2} X_{a}^{\prime}}\left[-\frac{1}{2 \pi}+\frac{X^{3 / 2}}{X_{a}^{\prime} H^{2}}\left(\frac{H_{a}^{\prime} H}{\pi X^{1 / 2}}+\frac{4 B}{a^{4}}\right)\right]^{-1}
$$




$$
c_{S}^{2}=\frac{1}{32 \pi^{2} w_{2}^{2} Q_{S}}\left(w_{2} H-\dot{w}_{2}-4 \pi w_{2}^{2}\right)=\frac{1}{32 \pi^{2} Q_{S}}\left[-4 \pi+H\left(\frac{a}{w_{2}}\right)_{a}^{\prime}\right],
$$

where

$$
w_{2}=\frac{H}{2}\left[\frac{1}{2 \pi}-\frac{X^{3 / 2}}{X_{a}^{\prime} H^{2}}\left(\frac{H_{a}^{\prime} H}{\pi X^{1 / 2}}+\frac{4 B}{a^{4}}\right)\right], B \equiv \varepsilon C_{0} \sqrt{2} .
$$

\section{RECONSTRUCTION EXAMPLES}

In this section, we will show reconstruction examples of the gravitating scalar field theories for various laws of the Universe expansion. Next, the theories will be tested for the absence of pathologies in the region of applicability of cosmological models.

\section{A. Power-law expanding Universe}

Now let's consider an expanding power-law Universe:

$$
a(t) \sim t^{n} \text { or } H \sim \frac{1}{a^{3 \gamma / 2}}, \gamma \neq 0 .
$$

In GR, the source of this law can be the perfect fluid:

$$
p=(\gamma-1) \rho, \rho=\frac{\rho_{0}}{a^{3 \gamma}},
$$

where $w=\gamma-1$ is the EoS parameter. Such functions 24) describe cosmological epochs with the radiation $(\gamma=4 / 3)$ or matter dominated $(\gamma=1)$. We define the input functions $H(a), X(a)$ :

$$
H=\sqrt{\frac{8 \pi \rho_{0}}{3}} \cdot \frac{1}{a^{3 \gamma / 2}}, X=\frac{A^{6(\gamma-1)}}{a^{6(\gamma-1)}}, A=\text { const }>0, \gamma \neq 1 .
$$

From equalities 12 and 13 we obtain

$$
\begin{gathered}
G_{2}=X^{\frac{\gamma}{2(\gamma-1)}} \cdot \frac{\rho_{0}}{A^{3 \gamma}}\left(-1+\frac{B A^{3(\gamma-1)}}{\rho_{0}}\right), \\
G_{3}=X^{\frac{2-\gamma}{4(\gamma-1)}} \cdot \frac{\varepsilon}{A^{3 \gamma / 2}(2-\gamma)} \sqrt{\frac{\rho_{0}}{12 \pi}} \cdot\left(\gamma-\frac{B A^{3(\gamma-1)}}{\rho_{0}}\right)+\text { const }, \gamma \neq 2 .
\end{gathered}
$$

Conditions for absence of ghosts and Laplacian instabilities

$$
\begin{gathered}
c_{S}^{2}=\frac{\rho_{0}}{3 B A^{3 \gamma-3}}\left(\gamma(3 \gamma-2)-\frac{B A^{3 \gamma-3}}{\rho_{0}}\right) \geq 0, \\
Q_{S}=\frac{3 A^{3 \gamma-3} B}{8 \pi \rho_{0}\left(\gamma-2+\frac{B A^{3 \gamma-3}}{\rho_{0}}\right)}>0
\end{gathered}
$$

are satisfied in two cases. Firstly, for $B>0$ :

$$
2-\gamma<\frac{B A^{3 \gamma-3}}{\rho_{0}} \leq \gamma(3 \gamma-2)
$$

The necessary condition for the fulfillment of (31) has the form: $\omega<-5 / 3, \omega>0$. Secondly, for $B<0$ :

$$
\gamma-2<\frac{|B| A^{3 \gamma-3}}{\rho_{0}} \leq \gamma(2-3 \gamma)
$$

The necessary condition for the fulfillment of 32 has the form: $-1<\omega<-1 / 3$. This model will not describe pressureless dark matter $(\gamma=1)$. Below we present a model with this substance. We observe that the conditions for absence of ghosts and Laplacian instabilities are always satisfied $(a(t)>0)$, and thus the scenario at hand is free from pathologies of the scalar sector at both background and perturbative level. Thus, the KGB theory can describe power-law expanding Universes by means of a nonzero scalar charge $(B \neq 0)$ and without other matter. 


\section{B. Exponential-law expanding Universe}

Here we will consider a dark energy equation of state $p=-\rho$. The Hubble parameter has the constant value

$$
H=\sqrt{\frac{8 \pi \rho_{0}}{3}}=\text { const, } a=a_{i} \exp (H t) .
$$

We take the kinetic density $X(a)$ in the form

$$
X=A^{2} a, A=\text { const }>0 .
$$

The two conditions (21), 222 for absence of pathologies in the scalar perturbations become

$$
Q_{S}=\frac{3}{8 \pi} \cdot \frac{|d|}{|d|+a^{5 / 2}}>0, c_{S}^{2}=\frac{\frac{a^{5 / 2}}{2}-\frac{|d|}{3}}{|d|+a^{5 / 2}} \geq 0
$$

where it is supposed $d \equiv \frac{3 A B}{\rho_{0}}<0(B<0)$. The condition $Q_{S}>0$ is satisfied for any values $a \geq 0$. The inequality $c_{S}^{2}>0$ holds for $a \geq a_{*}=\left(\frac{2|d|}{3}\right)^{2 / 5}$. Assuming that the gravity theory without quantum consideration works since the Planck times $t \gtrsim t_{p}$, it is natural to put $a_{*}=a_{p}=a\left(t_{p}\right)$. As the constant $\rho_{0}$, take the Planck density $\rho_{0}=\rho_{p}$. The profile of $c_{S}^{2}$ is shown in Fig. 11.

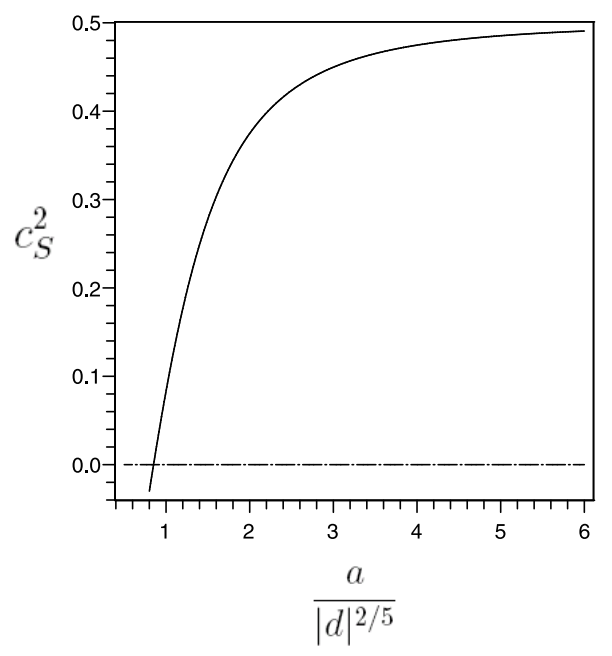

FIG. 1: Profile of the sound speed squared $c_{S}^{2}$.

From equalities 12 and 13 we obtain

$$
G_{2}=-\rho_{p}\left(1+\frac{|d| A^{5}}{3 X^{5 / 2}}\right), G_{3}=\frac{\varepsilon|d| A^{5} \sqrt{\rho_{p}}}{12 \sqrt{3 \pi} X^{3}}+\text { const } .
$$

The proposed model can describe the early inflation of the Universe since the Planck time without pathologies at the perturbative level (35). The area with pathology is "hidden" behind the Planck boundary $t=t_{p}$, where the gravity theory without quantum consideration has a dubious status. 


\section{Unification of dark matter - dark energy}

In $\Lambda$ CDM paradigm, the description of the transition to late the Universe acceleration is obtained by the usual consideration of a pressureless dark matter (DM) with the EoS parameter $w_{m}=0$ and a cosmological constant with the EoS parameter $w_{\Lambda}=-1$ :

$$
\frac{3 H^{2}}{8 \pi}=\rho=\rho_{d e}+\frac{\rho_{d m}}{a^{3}} ; \rho_{d e}, \rho_{d m}=\text { const },
$$

where $a\left(t_{0}\right)=1, t_{0}$ - the present moment of time. The deceleration parameter (DP)

$$
q(a)=\frac{d}{d t}\left(\frac{1}{H}\right)-1=\frac{a_{*}^{3}-a^{3}}{2 a_{*}^{3}+a^{3}}
$$

changes sign. There are two phases: $0 \leq a \leq a_{*}$ and $a>a_{*}$, where

$$
q\left(a_{*}\right)=0, a_{*}=\left(\frac{\rho_{d m}}{2 \rho_{d e}}\right)^{1 / 3}=\left(\frac{\Omega_{d m}}{2 \Omega_{d e}}\right)^{1 / 3} .
$$

Here, $\Omega_{d m}, \Omega_{d e}$ - the dimensionless density parameters. In the first phase there is no acceleration $(q \geq 0)$, and the second one is characterized by the Universe acceleration $(q<0)$.

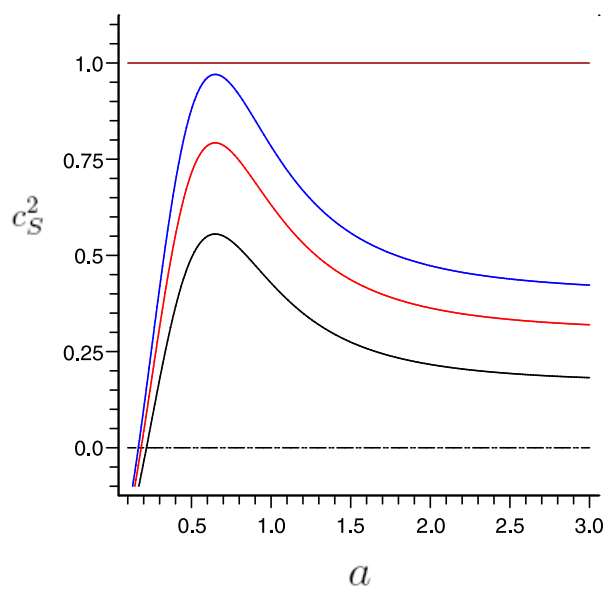

FIG. 2: Profiles of the sound speeds squared $c_{S}^{2}$ for $a_{*}=0.555$ and $\alpha=0.75 ; 0.95 ; 1.1$ (black, red, blue). The value $a_{*}$ corresponds a inflexion point $(q=0)$. The sound speed squared is positive, starting with small values of the scale factor $a$.

We will present a specific model, which presents a unified description of the DM and DE sectors. We define the input functions $H(a), X(a)$ :

$$
H=\sqrt{\frac{8 \pi}{3}\left(\rho_{d e}+\frac{\rho_{d m}}{a^{3}}\right)}, X=\rho_{d e}+\frac{\rho_{d m}}{a^{3}} .
$$

From equalities 12 and 13 we obtain

$$
G_{2}=-X-\frac{\rho_{d e} B X^{1 / 2}}{\rho_{d m}}+\frac{B X^{3 / 2}}{\rho_{d m}},
$$




$$
G_{3}=\frac{\varepsilon}{4 \rho_{d m} \sqrt{3 \pi}}\left(\rho_{d m} \ln \frac{X}{X_{0}}-2 B X^{1 / 2}\right)+\text { const } .
$$

The two conditions (21), 22 for absence of pathologies in the scalar perturbations become

$$
\begin{gathered}
Q_{S}=\frac{3}{8 \pi}>0, \\
c_{S}^{2}=\frac{\rho_{d m}\left(\rho_{d e}+\frac{4 \rho_{d m}}{a^{3}}\right)}{3 B\left(\rho_{d e}+\frac{\rho_{d m}}{a^{3}}\right)^{3 / 2}}-\frac{1}{3}=\frac{2 \alpha}{3} \frac{1+\frac{8 a_{*}^{3}}{a^{3}}}{\left(1+\frac{2 a_{*}^{3}}{a^{3}}\right)^{3 / 2}}-\frac{1}{3} \geq 0,
\end{gathered}
$$

where $\alpha=\rho_{d e}^{1 / 2} \cdot a_{*}^{3} / B$. It is assumed that $B>0$. There are values of the parameter $\alpha$ for which, starting from some value of the scale factor $a$, the stability condition 44 is fulfilled. The stability conditions are violated at small values of $a$. However, for $a \sim 0$, model (37) is not valid, and therefore the question of stability at small $a$ is not important. The profile of $c_{S}^{2}$ is shown in Fig. 2 .

Thus, the KGB theory can describe the late transitional Universe by means of a nonzero scalar charge $(B \neq 0)$ and without other matter.

\section{Post-inflationary transition to the radiation-dominated phase}

A fluid with the equation of state

$$
p=\frac{\rho}{3}-\mu \rho^{2}, \mu>0
$$

describes the transition from the inflationary era to the radiation era in the the early Universe 33. The linear equation of state $p=\rho / 3$ corresponds to radiation. The polytropic equation of state $p=-\mu \rho^{2}$ may be due to Bose-Einstein condensates with repulsive $(\mu<0)$ or attractive $(\mu>0)$ self-interaction, or have another origin. We will construct a scalar analogue of the theory 45 .

We write the DP as follows

$$
q(\rho)=\frac{\rho+3 p}{2 \rho}=1-\frac{3 \mu \rho}{2}=1-\frac{\rho}{\rho_{d}},
$$

where the parameter $\rho_{d}$ corresponds a inflexion point:

$$
q\left(\rho_{d}\right)=0, \rho_{d}=\frac{2}{3 \mu} .
$$

The Universe is accelerating when $\rho>\rho_{d}$ and decelerating when $\rho \leq \rho_{d}$. The "equation of continuity"

$$
\dot{\rho}+3 H(\rho+\rho)=0
$$

can be integrated into $\rho=\frac{2 \rho_{d}}{a^{4}+1}$, where we assume that $a=a_{d}=1$ corresponds to the end of inflation. When $a \ll 1$, the density $\rho$ takes the maximum value $2 \rho_{d}$. The Planck density $\rho_{p}$ will be determined so that it approximately corresponds to he maximum value: $\rho_{p} \approx 2 \rho_{d}$. Thus the density will take the form

$$
\rho=\frac{\rho_{p}}{a^{4}+1}
$$

which gives the Hubble parameter

$$
H^{2}=\frac{8 \pi \rho_{p}}{3} \cdot \frac{1}{a^{4}+1}
$$

During the inflationary phase $(a \ll 1)$, the parameter $H$ has an approximately constant value $H \simeq \sqrt{\frac{8 \pi \rho_{p}}{3}}$. After the inflation $(a \gg 1)$, the Universe enters in the radiation era: $H \simeq \sqrt{\frac{8 \pi \rho_{p}}{3 a^{4}}}$. This model was considered in the work 33 . 
We chose the Hubble parameter $H$ according to the task. We take the kinetic density $X(a)$ in the form

$$
X(a)=\frac{A^{2}}{a^{2}}, A=\text { const }>0 .
$$

The two conditions 21, 22 for absence of pathologies in the scalar perturbations become

$$
\begin{gathered}
Q_{S}=\frac{3 d \cdot\left(1+a^{4}\right)^{2}}{8 \pi\left[2 a^{4}\left(1-a^{4}\right)+d \cdot\left(1+a^{4}\right)^{2}\right]}>0, \\
c_{S}^{2}=\frac{-d^{2} \cdot\left(1+a^{4}\right)^{4}+2 d \cdot a^{4}\left(1+a^{4}\right)^{2}\left(5 a^{4}+3\right)+16 a^{12}\left(3-a^{4}\right)}{3 d \cdot\left(1+a^{4}\right)^{2}\left[2 a^{4}\left(1-a^{4}\right)+d \cdot\left(1+a^{4}\right)^{2}\right]} \geq 0,
\end{gathered}
$$

where $d \equiv \frac{3 A B}{\rho_{p}}$. They have the following limits:

$$
\begin{gathered}
\frac{3}{8 \pi} \leftarrow Q_{S} \rightarrow \frac{3 d}{8 \pi(d-2)} \text { as } 0 \leftarrow a \rightarrow+\infty, \\
-\frac{1}{3} \leftarrow c_{S}^{2} \rightarrow \frac{8-d}{3 d} \text { as } 0 \leftarrow a \rightarrow+\infty, d \neq 2 .
\end{gathered}
$$

In case $d>2$, the condition $Q_{S}>0$ is satisfied for any values $a \geq 0$. There are values of the parameter $d$ for which, starting from some value of the scale factor $a$, the stability condition 52 is fulfilled. The Fig. 3 shows that the sound speeds squared become negative at early times, $a \lesssim 0.82815$ (for $d=8 / 3$ ). The transition point $a_{d}=1$ to unaccelerated expansion of the Universe is in the region of stability of the model for $d=8 / 3$.

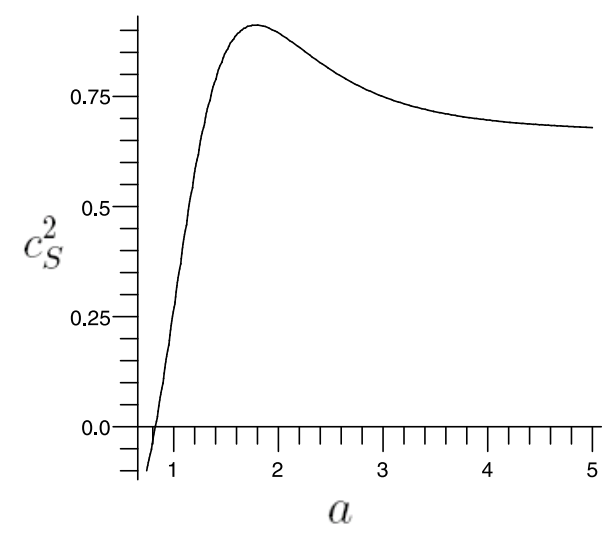

FIG. 3: Profile of the sound speed squared $c_{S}^{2}$ for $d=8 / 3$. The value $a=a_{d}=1$ corresponds a inflexion point $(q=0)$. The sound speed squared is positive for $a \gtrsim 0.82815$.

From equalities 12 and 13 we obtain

$$
G_{2}=\frac{\rho_{p} \cdot X^{2}\left(d \cdot X^{2}+(d-3) A^{4}\right)}{3 A^{4}\left(A^{4}+X^{2}\right)}
$$




$$
G_{3}=\frac{\varepsilon}{2} \sqrt{\frac{\rho_{p}}{3 \pi}} \int^{X} d X \cdot\left[\frac{A^{4}}{X^{1 / 2}\left(A^{4}+X^{2}\right)^{3 / 2}}-\frac{d \cdot\left(A^{4}+X^{2}\right)^{1 / 2}}{4 A^{4} X^{1 / 2}}\right]+\text { const } .
$$

The model is unstable for $a \ll 1$. During the radiation era $(a \gg 1)$, the functions $G_{2}, G_{3}$ have approximately view:

$$
G_{2} \simeq \frac{\rho_{p}(d-3) \cdot X^{2}}{3 A^{4}}, G_{3} \simeq \frac{\varepsilon(4-d)}{4 A^{2}} \cdot \sqrt{\frac{\rho_{p} \cdot X}{3 \pi}}+\text { const }
$$

\section{CONCLUSION}

The HG theory action density (3) contains arbitrary functions $G_{i}(X, \phi), i=2,3,4,5$. The standard algorithm assumes setting the functions $G_{i}(X, \phi)$, obtaining a solution for the scale factor $a(t)$ and the scalar field $\phi(t)$, and analyzing them for compliance with the observed data. In HG theory, as a rule, this approach requires considerable effort and the involvement of numerical methods for solving a differential equations system. The search result for a solution is not predictable. Another way is the reconstruction method. The evolution scenario of the Universe is set a priori. It serves to limit and determine the parameters of the studied modified gravity theory. In this article, we have proposed a way to reconstruct the KGB theory with shiftsymmetric based on the Hubble evolution in the flat FRW spacetimes. The dynamic solution $H(a)$ is provided by a nonzero scalar charge associated with the shift symmetry. In the described method, the choice of the Hubble parameter $H(a)$ does not uniquely determine the scalar theory. To complete the construction, we need to set the kinetic density $X(a)$. This freedom is used to eliminate the instability of perturbations in the range of applicability of the considered cosmological models. The process of selecting function $X(a)$ is heuristic. We have illustrated this method for models: the perfect fluid, the unified description dark energy-dark matter, the post-inflationary transition to the radiation-dominated phase.

We have succeeded in constructing a model of perfect fluid $(\omega \neq-1)$ that is stable throughout the expansion history. Other models contain the instability on a set of values $a \in\left[0, a_{1}\right)$. This set is not included in the range of applicability of cosmological models (33) and (37), therefore the question of stability is not important on $\left[0, a_{1}\right)$ for these models. The post-inflationary transition model $(45)$ is stable around the transition point $a_{d}$ and in the future.

\section{Acknowledgments}

This work is supported by the Russian Foundation for Basic Research (Grant No. 19-52-15008).

[1] B. P. Abbott, R. Abbott, T. Abbott, M. Abernathy, F. Acernese, K. Ackley et al.: Phys. Rev. Lett. 116, 061102 (2016)

[2] B. P. Abbott, R. Abbott, T. Abbott, M. Abernathy, F. Acernese, K. Ackley et al.: Phys. Rev. Lett. 116, 241103 (2016)

[3] L. Scientific, B. Abbott, R. Abbott, T. Abbott, F. Acernese, K. Ackley et al.: Phys. Rev. Lett. 118, 221101 (2017)

[4] B. P. Abbott, R. Abbott, T. Abbott, F. Acernese, K. Ackley, C. Adams et al.: Phys. Rev. Lett. 119, 141101 (2017)

[5] L. S. Collaboration and V. Collaboration: Phys. Rev. Lett. 119, 161101 (2017)

[6] S. Deser and P. van Nieuwenhuizen: Phys. Rev. Lett. 32, 245 (1974)

[7] S. Hawking and R. Laflamme: Physics Letters B 209, 39 (1988)

[8] M. Martellini: Phys. Rev. Lett. 51, 152 (1983)

[9] J. F. Donoghue: Phys. Rev. D 50, 3874 (1994)

[10] G.W. Horndeski: Int. J. Theor. Phys. 10, 363 (1974)

[11] M. Ostrogradski: Mem. Ac. St. Petersbourg VI 4, 385 (1850)

[12] R. P. Woodard: Scholarpedia 10, 32243 (2015); arXiv preprint arXiv: 1506.02210 (2015)

[13] E.J. Copeland, M. Sami and S. Tsujikawa, Dynamics of dark energy, Int. J. Mod. Phys. D 15, 1753 (2006)

[14] A. Joyce, B. Jain, J. Khoury and M. Trodden, Beyond the Cosmological Standard Model, Phys. Rept. 568, 1 (2015)

[15] C. Armendariz-Picon, V. Mukhanov and P. J. Steinhardt: Phys. Rev. D 63, 103510 (2001)

[16] S. Tsujikawa: Classical and Quantum Gravity 30, 214003 (2013)

[17] C. Deffayet, O. Pujolas, I. Sawicki and A. Vikman: Journal of Cosmology and Astroparticle Physics 2010, 026 (2010)

[18] T. Kobayashi: arXiv preprint arXiv:1901.07183 (2019)

[19] S.V. Sushkov: Phys. Rev. D 80, 103505 (2009)

[20] S.A. Appleby, A. De Felice and E.V. Linder: JCAP 10060 (2012)

[21] T. P. Sotiriou and S.-Y. Zhou: Phys. Rev. D 90124063 (2014)

[22] E. Babichev, C. Charmousis and A. Lehebel: Classical and Quantum Gravity 33154002 (2016)

[23] A. Maselli, H. O. Silva, M. Minamitsuji and E. Berti: Phys. Rev. D 92, 104049 (2015) 
[24] A. I. Vainshtein: Phys. Lett. B 39, 393 (1972)

[25] N. Chow and J. Khoury: Phys. Rev. D 80, 024037 (2009)

[26] A. De Felice, R. Kase and S. Tsujikawa: Phys. Rev. D 85, 044059 (2012)

[27] T. Baker, E. Bellini, P. G. Ferreira, M. Lagos, J. Noller and I. Sawicki: Phys. Rev. Lett. 119251301 (2017)

[28] D. Bettoni, J. M. Ezquiaga, K. Hinterbichler and M. Zumalacarregui: Phys. Rev. D 95, 084029 (2017)

[29] A. De Felice and S. Tsujikawa: JCAP 1202, 007 (2012)

[30] S. A. Appleby and E. V. Linder: JCAP 1203, 043 (2012)

[31] T. Kobayashi, M. Yamaguchi and J. Yokoyama: Prog. Theor. Phys. 126, 511 (2011)

[32] R. C. Bernardo and I. Vega: JCAP 1910, 058 (2019)

[33] P.H. Chavanis: Eur. Phys. J. Plus, 129, 38 (2014)

[34] D.A. Easson, I. Sawicki and A. Vikman: JCAP 1111, 021 (2011) arXiv:1109.1047

[35] T. Helpin and M.S. Volkov: JCAP 2001, 044 (2020) arXiv:1906.07607 